\title{
Automatic continuity of homomorphisms between topological semigroups
}

\author{
Taras Banakh • Iryna Pastukhova
}

Received: 8 October 2013 / Accepted: 29 May 2014 / Published online: 10 December 2014

(C) The Author(s) 2014. This article is published with open access at Springerlink.com

\begin{abstract}
According to an old theorem of Yeager (Trans Am Math Soc 215:253267, 1976), a homomorphism $h: X \rightarrow Y$ between compact Hausdorff topological Clifford semigroups is continuous if and only if for every subgroup $H \subset X$ and every subsemilattice $E \subset X$ the restrictions $h \mid H$ and $h \mid E$ are continuous. In this paper we extend this Yeager result beyond the class of compact topological Clifford semigroups.
\end{abstract}

Keywords Continuous homomorphism - Topological inverse semigroup . Topological Clifford semigroup · Topological semilattice

\section{Motivation and principal problem}

This paper was motivated by the following old result of Yaeger [13].

Theorem 1.1 [13] A homomorphism $h: X \rightarrow Y$ between compact topological Clifford semigroups is continuous if and only if for any subgroup $H \subset X$ and any subsemilattice $E \subset X$ the restrictions $h \mid H$ and $h \mid E$ are continuous.

In this paper we shall extend this Yeager's theorem beyond the class of compact topological Clifford semigroups. It will be convenient to use the following notion.

Communicated by Jimmie D. Lawson.

T. Banakh · I. Pastukhova

Ivan Franko National University of Lviv, Lviv, Ukraine

I. Pastukhova

e-mail: irynkapastukhova@gmail.com

T. Banakh $(\bowtie)$

Jan Kochanowski University in Kielce, Kielce, Poland

e-mail: t.o.banakh@gmail.com 
Definition 1.2 A homomorphism $h: X \rightarrow Y$ between topological semigroups is called EH-continuous if

- the restriction $h \mid E_{X}$ to the set of idempotents $E_{X}=\{e \in X: e e=e\}$ of $X$ is continuous;

- for each subgroup $H \subset X$ the restriction $h \mid H$ is continuous.

So, in terms of $E H$-continuity, Theorem 1.1 says that each $E H$-continuous homomorphism between compact Hausdorff topological Clifford semigroups is continuous. This Yeager's theorem suggests the following problem addressed in this paper.

Problem 1.3 Find conditions on topological semigroups $X, Y$ guaranteeing that each $E H$-continuous homomorphism $h: X \rightarrow Y$ is continuous.

We shall answer this problem in Sects. 6 and 7 after some preliminary work done in Sects. 2-5.

\section{Preliminaries}

In this section we collect some known information related to topological semigroups and topological spaces.

\subsection{Semigroups}

A semigroup is a non-empty set $S$ endowed with an associative binary operation. A semigroup $S$ is called inverse if for each element $x \in S$ there is a unique element $x^{-1} \in S$ such that $x x^{-1} x=x$ and $x^{-1} x x^{-1}=x^{-1}$. An inverse semigroup $S$ is called a Clifford semigroup if $x x^{-1}=x^{-1} x$ for all $x \in S$. For a semigroup $S$ by $E_{S}=\{e \in S: e e=e\}$ we denote the set of idempotents of $S$. For each idempotent $e \in E_{S}$ by

$$
H_{e}=\{x \in S: \exists y \in S x y=e=y x, x e=x=e x, y e=y=e y\}
$$

we denote the maximal subgroup of $S$ containing the idempotent $e$. It is known that each Clifford semigroup $S$ decomposes into the disjoint union $\bigcup_{e \in E_{S}} H_{e}$ of maximal groups $H_{e}=\left\{x \in S: x x^{-1}=e=x^{-1} x\right\}$ parameterized by idempotents $e$ of $S$.

A semigroup $S$ is regular if $x \in x S x$ for each $x \in S$. It is known [10, II.1.2] that a semigroup $S$ is inverse if and only if $S$ is regular and the subset $E_{S}$ is a commutative subsemigroup of $S$. In this case $E_{S}$ is the maximal semilattice of $S$. A semilattice is a commutative semigroup of idempotents. Each semilattice $E$ carries a natural partial order $\leq$ defined by $x \leq y$ iff $x y=x$. In this partial order the semilattice operation coincides with the operation of minimum.

A homomorphism between semigroups $X, Y$ is a function $h: X \rightarrow Y$ preserving the operation in the sense that $h(x \cdot y)=h(x) \cdot h(y)$ for all $x, y \in X$. The uniqueness of the inverse element in an inverse semigroup implies that each homomorphism $h: X \rightarrow Y$ between inverse semigroups preserves the inversion in the sense that $h\left(x^{-1}\right)=h(x)^{-1}$ for all $x \in X$. 


\subsection{Unosemigroups and their unomorphisms}

By a left unit operation on a semigroup $S$ we understand a unary operation $\lambda_{S}: S \rightarrow S$ such that $\lambda_{S}(x) \cdot x=x$ for all $x \in S$. A left unosemigroup is a semigroup $S$ endowed with a left unit operation $\lambda_{S}: S \rightarrow S$. A left unosemigroup $S$ is called $\lambda$-regular if for each $x \in S$ there is $x^{*} \in S$ such that $\lambda_{S}(x)=x x^{*}$. In this case the element $\lambda_{S}(x)=$ $x x^{*}$ is an idempotent because $\lambda_{S}(x) \cdot \lambda_{S}(x)=\lambda_{S}(x) x x^{*}=x x^{*}=\lambda_{S}(x)$. So, for each $\lambda$-regular left unosemigroup $S$ we get $\lambda_{S}(S) \subset E_{S}$. Each $\lambda$-regular unosemigroup is a regular semigroup, and conversely, each regular semigroup $S$ can be endowed with a left unit operation $\lambda_{S}: S \rightarrow S$ turning it into a $\lambda$-regular unosemigroup.

By a unomorphism between left unosemigroups $\left(X, \lambda_{X}\right)$ and $\left(Y, \lambda_{Y}\right)$ we understand a semigroup homomorphism $h: X \rightarrow Y$ preserving the left unit operation in the sense that $h \circ \lambda_{X}=\lambda_{Y} \circ h$.

By analogy we can define right versions of the above concepts. In particular, a right unosemigroup is a semigroup $S$ endowed with a right unit operation $\rho_{S}: S \rightarrow S$ such that $x \cdot \rho_{S}(x)=x$ for all $x \in S$. A right unosemigroup $S$ is $\rho$-regular if for every $x \in S$ there is $x^{*} \in S$ such that $\rho_{S}(x)=x^{*} x$. In this case $\rho_{S}(S) \subset E_{S}$. A unomorphism between right unosemigroups $\left(X, \rho_{X}\right)$ and $\left(Y, \rho_{Y}\right)$ is a semigroup homomorphism $h: X \rightarrow Y$ preserving the right unit operation in the sense that $h \circ \rho_{X}=\rho_{Y} \circ h$.

A unosemigroup is a semigroup $S$ endowed with a left unit operation $\lambda_{S}$ and a right unit operation $\rho_{S}$. A unosemigroup $S$ is regular if it is $\lambda$-regular and $\rho$-regular. A unomorphism between unosemigroups $\left(X, \lambda_{X}, \rho_{X}\right)$ and $\left(Y, \lambda_{Y}, \rho_{Y}\right)$ is a semigroup homomorphism $h: X \rightarrow Y$ preserving the unit operations in the sense that $h \circ \lambda_{X}=$ $\lambda_{Y} \circ h$ and $h \circ \rho_{X}=\rho_{Y} \circ h$.

Each inverse semigroup $S$ endowed with the left unit operation $\lambda_{S}: S \rightarrow S$, $\lambda_{S}: x \mapsto x x^{-1}$, and the right unit operation $\rho_{S}: S \rightarrow S, \rho_{S}: x \mapsto x^{-1} x$, carries a canonical structure of a regular unosemigroup. Each homomorphism between inverse semigroups is a unomorphism of the corresponding unosemigroups.

\subsection{Topological semigroups and unosemigroups}

Now we recall the topological versions of the above algebraic notions. A topological semigroup is a semigroup $S$ endowed with a topology making the semigroup operation $: S \times S \rightarrow S$ continuous. A topological inverse (Clifford) semigroup is an inverse (Clifford) semigroup endowed with a topology making the semigroup operation $\cdot: S \times$ $S \rightarrow S$ and the inversion operation ( $)^{-1}: S \rightarrow S$ continuous.

A topological unosemigroup is a topological semigroup $S$ endowed with a continuous left unit operation $\lambda_{S}$ and a continuous right unit operation $\rho_{S}$. By analogy we can define topological left unosemigroups and topological right unosemigroups.

In the proof of Theorem 3.2 we shall use the following property of $\lambda$-regular topological left unosemigroups.

Proposition 2.1 If a topological left unosemigroup $\left(S, \lambda_{S}\right)$ is $\lambda$-regular, then for any idempotente $\in S$ and any point $x \in S$ withe $\cdot \lambda_{S}(x)=e$ the right shift $s_{x}: H_{e} \rightarrow H_{e} x$, $s_{x}: z \mapsto z x$, is a homeomorphism. 
Proof Since $\left(S, \lambda_{S}\right)$ is $\lambda$-regular, $\lambda_{S}(x)=x x^{*}$ for some element $x^{*} \in S$. Consider the right shift $s_{x^{*}}: S \rightarrow S, s_{x^{*}}: z \mapsto z x^{*}$, and observe that for every element $z$ of the maximal subgroup $H_{e}$, we get $s_{x^{*}} \circ s_{x}(z)=z x x^{*}=z \cdot \lambda_{S}(x)=z e \cdot \lambda_{S}(x)=z e=z$. This implies that the restriction $s_{x^{*}} \mid H_{e} x: H_{e} x \rightarrow H_{e}$ is a continuous map, inverse to $s_{x}$. So, $s_{x}: H_{e} \rightarrow H_{e} x$ is a homeomorphism.

\subsection{Ditopological unosemigroups}

For two subsets $A, B$ of a semigroup $S$ consider the subsets

$$
\begin{aligned}
& B^{[-1]} A=\{y \in S: \exists b \in B \exists a \in A \quad b y=a\} \text { and } A B^{[-1]} \\
& \quad=\{x \in S: \exists a \in A \exists b \in B \quad a=x b\}
\end{aligned}
$$

which can be thought as the results of left and right division of $A$ by $B$ in the semigroup $S$.

A topological left unosemigroup $\left(S, \lambda_{S}\right)$ is called a ditopological left unosemigroup if for each $x \in S$ and neighborhood $O_{x} \subset S$ there are neighborhoods $W_{\lambda_{S}(x)} \subset \lambda_{S}(S)$ and $U_{x} \subset S$ of the points $\lambda_{S}(x)$ and $x$, respectively, such that

$$
\left(W_{\lambda_{S}(x)}^{[-1]} U_{x}\right) \cap \lambda_{S}^{-1}\left(W_{\lambda_{S}(x)}\right) \subset O_{x} .
$$

By analogy we can introduce a right version of this notion. Namely, a ditopological right unosemigroup is a topological right unosemigroup $\left(S, \rho_{S}\right)$ such that for each $x \in X$ and neighborhood $O_{x} \subset S$ there are neighborhoods $W_{\rho_{S}(x)} \subset \rho_{S}(S)$ and $U_{x} \subset S$ of the points $\rho_{S}(x)$ and $x$, respectively, such that

$$
\left(U_{x} W_{\rho_{S}(x)}^{[-1]}\right) \cap \rho_{S}^{-1}\left(W_{\rho_{S}(x)}\right) \subset O_{x} .
$$

A topological unosemigroup $\left(S, \lambda_{S}, \rho_{S}\right)$ is called a ditopological unosemigroup if $\left(S, \lambda_{S}\right)$ is a ditopological left unosemigroup and $\left(S, \rho_{S}\right)$ is a ditopological right unosemigroup.

Ditopological unosemigroups were introduced in [1] and studied in [1] and [2]. In [1] it was shown that the class of ditopological unosemigroups contains all compact Hausdorff topological unosemigroups, is closed under taking subunosemigroups, Tychonoff, reduced, and semidirect products, and has many other nice properties.

\subsection{Ditopological inverse semigroups}

A topological inverse (Clifford) semigroup $S$ is called a ditopological inverse (Clifford) semigroup if $S$, endowed with its canonical left and right unit operations, is a ditopological unosemigroup. By [1], a topological inverse semigroup $S$ is ditopological if and only if $\left(S, \lambda_{S}\right)$ is a ditopological left unosemigroup if and only if $\left(S, \rho_{S}\right)$ is a ditopological right unosemigroup. 
The class of ditopological inverse semigroups contains all compact Hausdorff topological inverse semigroups, all topological groups, all topological semilattices, and is closed under taking inverse subsemigroups and Tychonoff products, see [1]. So, this is a class nicely extending the class of compact topological inverse semigroups and many results known for compact topological inverse semigroups extend to ditopological inverse semigroups, see [2]. Let us write down one of these facts for future references.

Proposition 2.2 Each compact Hausdorff topological inverse semigroup is ditopological.

\subsection{General topology}

In this subsection we recall some information from General Topology. For a subset $A$ of a topological space $X$ its closure will be denoted by $\mathrm{cl}_{X}(A)$, or $\operatorname{cl}(A)$ or just $\bar{A}$ (if the space $X$ is clear from the context).

A topological space $X$ is called

- Fréchet-Urysohn (or briefly, Fréchet) if for each set $B \subset X$ and a point $x \in \bar{B}$ in its closure the set $B$ contains a sequence convergent to $x$;

- sequential if each non-closed subset $B \subset X$ contains a sequence convergent to a point $x \in \bar{B} \backslash B$.

It is clear that each metrizable topological space is Fréchet and each Fréchet space is sequential. By $[8,2.4 . \mathrm{G}]$ a space $X$ is sequential if and only if each subspace of $X$ is Fréchet.

A topological space $X$ is called

- countably compact if each sequence in $X$ has an accumulation point;

- Čech-complete if $X$ is Tychonoff and $X$ is a $G_{\delta}$-set in its Stone-Čech compactification $\beta X$;

- Baire if the intersection $\bigcap_{n \in \omega} U_{n}$ of any sequence $\left(U_{n}\right)_{n \in \omega}$ of open dense subsets of $X$ is dense in $X$;

- hereditarily Baire if each closed subspace of $X$ is Baire.

By Hurewicz's theorem [9], a metrizable space $X$ is hereditarily Baire if and only if $X$ does not contain a closed subspace homeomorphic to the space $\mathbb{Q}$ of rational numbers.

\subsection{Two topological games}

It is known that regular countably compact spaces and Čech-complete spaces are hereditarily Baire. In this subsection we consider two classes of spaces containing all countably compact spaces and all Čech-complete spaces and contained in the class of hereditarily Baire spaces. These spaces are defined with help of (a Bouziad's modification of) the classical strong Choquet game [6].

The strong Choquet game on a topological space $X$ is played by two players $P$ and $O$. The player $P$ starts the game choosing an open set $P_{0} \subset X$ and a point $p_{0} \in P_{0}$, 
and the player $O$ responds selecting a neighborhood $O_{0} \subset P_{0}$ of $p_{0}$. At the $n$th inning the player $P$ selects an open set $P_{n} \subset O_{n-1}$ and a point $p_{n} \in P_{n}$ and the player $O$ responds selecting a neighborhood $O_{n} \subset P_{n}$ of $p_{n}$. At the end of the game, the player $O$ is declared the winner if the intersection $\bigcap_{n=1}^{\infty} \mathrm{cl}_{X}\left(O_{n}\right)$ is not empty.

A topological space $X$ is called

- Choquet-complete if the player $O$ has a winning strategy in the strong Choquet game on $X$;

- Choquet-saturated if the player $P$ has no winning strategy in the strong Choquet game on $X$.

The following two theorems (due to Choquet [6] and Telgárski [11], [12], Debs [7]) characterize metrizable spaces, which are Choquet-complete or Choquet-saturated, respectively.

Theorem 2.3 (Choquet) A (metrizable) Tychonoff space is Choquet-complete if (and only if) it is Čech-complete.

Theorem 2.4 (Telgársky, Debs) A (metrizable) regular space is Choquet-saturated (if and) only if it is hereditarily Baire.

Next, we consider a modification of the strong Choquet game suggested by A. Bouziad [3]. Like the strong Choquet game, the Bouziad game is played on a topological space $X$ by two players, $P$ and $O$. The player $P$ starts the game choosing a point $p_{0} \in X$ and the player $O$ responds selecting a neighborhood $O_{0} \subset X$ of $p_{0}$. At the $n$th inning the player $P$ chooses a point $p_{n} \in O_{n-1}$ and the player $P$ responds by a neighborhood $O_{n}$ of $p_{n}$. At the end of the game the player $O$ is declared the winner if the sequence $\left(p_{n}\right)_{n=1}^{\infty}$ constructed by the player $P$ has an accumulation point in $X$.

A topological space $X$ is defined to be

- Bouziad-complete if the player $O$ has a winning strategy in the Bouziad game on $X$;

- Bouziad if the player $P$ has no winning strategy in the Bouziad game on $X$.

It follows from the definitions of the strong Choquet and Bouziad games that each Bouziad-complete space is Choquet-complete and each Bouziad space is Choquetsaturated. For metrizable spaces these implications can be reversed.

Proposition 2.5 A metrizable topological space $X$ is Bouziad-complete if and only if it is Choquet-complete if and only if $X$ is Čech-complete.

Proposition 2.6 A metrizable topological space $X$ is Bouziad if and only if it is Choquet-saturated if and only if $X$ is hereditarily Baire.

This proposition follows from Theorems 2.3 and 2.4 and the observation that for a decreasing sequence $O_{1} \supset O_{2} \supset \cdots$ of non-empty open subsets of a metric space $X$ with diam $O_{n} \rightarrow 0$ and any points $x_{n} \in O_{n}, n \in \mathbb{N}$, the intersection $\bigcap_{n=1}^{\infty} \bar{O}_{n}$ is not empty if and only if the sequence $\left(x_{n}\right)_{n \in \mathbb{N}}$ has an accumulation point in $X$. 
Thus for a Tychonoff topological space $X$ we have the following implications:

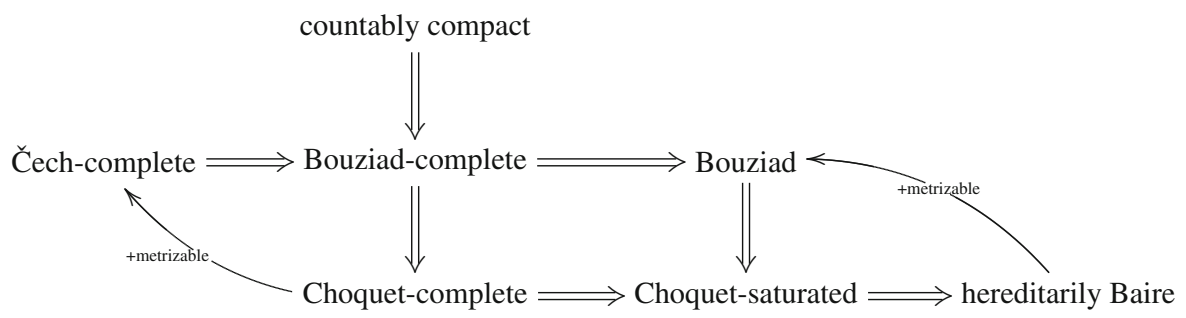

\section{$3 \mathrm{E} H$-Continuous unomorphisms between topological unosemigroups}

In this section we study the problem of automatic continuity of $E H$-continuous unomorphisms between topological unosemigroups.

Definition 3.1 We define a topological unosemigroup $(S, \lambda, \rho)$ to be group-refractive if for every non-closed set $B \subset S$ there is a point $x \in \bar{B} \backslash B$ such that for every neighborhood $O_{\lambda(x)} \subset \lambda(S)$ of the idempotent $\lambda(x)$ there are a subset $B_{x} \subset B$ and an idempotent $e \in O_{\lambda(x)}$ such that $x \in \operatorname{cl}\left(B_{x}\right), e \cdot \lambda(x)=e$ and $e B_{x} \rho(x) \subset H_{e} x$.

Theorem 3.2 If a $\lambda$-regular topological unosemigroup $X$ is group-refractive, then each EH-continuous unomorphism $h: X \rightarrow Y$ to a ditopological unosemigroup $Y$ is continuous.

Proof Assuming that some $E H$-continuous unomorphism $h: X \rightarrow Y$ is not continuous, we can find an open subset $O_{Y} \subset Y$ whose preimage $h^{-1}\left(O_{Y}\right)$ is not open in $X$. Then the set $B=X \backslash h^{-1}\left(O_{Y}\right)$ is not closed in $X$ and by the group-refractivity of $X$, there is a point $x \in \bar{B} \backslash B$ such that for every neighborhood $O_{\lambda_{X}(x)} \subset \lambda_{X}(X) \subset E_{X}$ of $\lambda_{X}(x)$ there are a subset $B_{x} \subset B$ and an idempotent $e \in O_{\lambda_{X}(x)}$ such that $x \in \operatorname{cl}_{X}\left(B_{x}\right)$, $e \cdot \lambda_{X}(x)=e$ and $e \cdot B_{x} \cdot \rho_{X}(x) \subset H_{e} \cdot x$. It follows from $x \in \bar{B} \backslash B$ that $x \in h^{-1}\left(O_{Y}\right)$ and hence $h(x) \in O_{Y}$.

Since the topological right unosemigroup $\left(Y, \rho_{Y}\right)$ is ditopological, for the point $y=h(x) \in Y$ and its open neighborhood $O_{Y} \subset Y$, there are open neighborhoods $W_{\rho_{Y}(y)} \subset \rho_{Y}(Y)$ and $U_{y} \subset O_{Y} \subset Y$ of the points $\rho_{Y}(y)$ and $y$, respectively, such that $\left(U_{y} W_{\rho_{Y}(y)}^{[-1]}\right) \cap \rho_{Y}^{-1}\left(W_{\rho_{Y}(y)}\right) \subset O_{Y}$.

Since the topological left unosemigroup $\left(Y, \lambda_{Y}\right)$ is ditopological, for the point $y=h(x) \in Y$ and its open neighborhood $U_{y} \subset Y$, there are open neighborhoods $W_{\lambda_{Y}(y)} \subset \lambda_{Y}(Y)$ and $V_{y} \subset U_{y} \subset Y$ of the points $\lambda_{Y}(y)$ and $y$, respectively, such that $\left(W_{\lambda_{Y}(y)}^{[-1]} V_{y}\right) \cap \lambda_{Y}^{-1}\left(W_{\lambda_{Y}(y)}\right) \subset U_{y}$.

Taking into account that $\lambda_{Y}(y) \cdot y \cdot \rho_{Y}(y)=y$, we can replace $W_{\lambda_{Y}(y)}$ and $W_{\rho_{Y}(y)}$ by smaller neighborhoods and additionally assume that $W_{\lambda_{Y}(y)} \cdot y \cdot W_{\rho_{Y}(y)} \subset V_{y}$.

Since the unomorphism $h$ preserves the left unary operation we have $h\left(\lambda_{X}(x)\right)=$ $\lambda_{Y}(y)$. The $\lambda$-regularity of the unary operation $\lambda_{X}$ implies that $\lambda_{X}(X) \subset E_{X}$. Then the continuity of the restriction $h \mid E_{X}$ yields an open neighborhood $W_{\lambda_{X}(x)} \subset \lambda_{X}(X)$ of $\lambda_{X}(x)$ such that $h\left(W_{\lambda_{X}(x)}\right) \subset W_{\lambda_{Y}(y)}$. By the choice of the point $x$, we can find a 
subset $B_{x} \subset B$ and an idempotent $e \in W_{\lambda_{X}(x)}$ such that $x \in \operatorname{cl}_{X}\left(B_{x}\right), e \cdot \lambda_{X}(x)=e$ and $e \cdot B_{x} \cdot \rho_{X}(x) \subset H_{e} \cdot x$.

Consider the idempotent $e^{\prime}=h(e) \in W_{\lambda_{Y}(y)}$ and observe that $e^{\prime} \cdot y \cdot \rho_{Y}(y) \in$ $W_{\lambda_{Y}(y)} \cdot y \cdot W_{\rho_{Y}(y)} \subset V_{y}$. Since the unomorphism $h$ is $E H$-continuous, its restriction $h \mid H_{e}$ to the maximal subgroup $H_{e} \subset X$ is continuous. Consequently, the idempotent $e$ has an open neighborhood $O_{e} \subset H_{e}$ such that $h\left(O_{e}\right) \subset W_{\lambda_{Y}(y)}$. By Proposition 2.1, the right shift $s_{x}: H_{e} \rightarrow H_{e} x$ is a homeomorphism, which implies that the set $O_{e} x=s_{x}\left(O_{e}\right)$ is an open neighborhood of the element ex in $H_{e} x$. Since the set

$$
\left.W_{x}=\left\{w \in X: \lambda_{X}\left(w \cdot \rho_{X}(x)\right) \in W_{\lambda_{X}(x)}\right)\right\} \cap\left\{w \in X: h \circ \rho_{X}(w) \in W_{\rho_{Y}(y)}\right\}
$$

is an open neighborhood of $x$, the intersection $B_{x} \cap W_{x}$ contains $x$ in its closure, which implies that $e x=e \cdot x \cdot \rho_{X}(x)$ lies in the closure of the set $e \cdot\left(B_{x} \cap W_{x}\right) \cdot \rho_{X}(x) \subset$ $H_{e} x$. Since $O_{e} x$ is a neighborhood of $e x$ in $H_{e} x$, there is a point $b \in B_{x}$ such that $e \cdot b \cdot \rho_{X}(x) \in O_{e} x$.

Consider the element $h(b) \in Y$ and observe that

$$
e^{\prime} \cdot h\left(b \cdot \rho_{X}(x)\right)=h\left(e \cdot b \cdot \rho_{X}(x)\right) \in h\left(O_{e} x\right)=h\left(O_{e}\right) \cdot h(x) \subset W_{\lambda_{Y}(y)} \cdot y \subset V_{y}
$$

and $e^{\prime}=h(e) \in h\left(W_{\lambda_{X}(x)}\right) \subset W_{\lambda_{Y}(y)}$ imply

$$
h\left(b \cdot \rho_{X}(x)\right) \in W_{\lambda_{Y}(y)}^{[-1]} V_{y} .
$$

On the other hand, the inclusion $b \in B_{x} \cap W_{x}$ and the definition of the set $W_{x}$ imply

$$
\lambda_{Y} \circ h\left(b \cdot \rho_{X}(x)\right)=h \circ \lambda_{X}\left(b \cdot \rho_{X}(x)\right) \in h\left(W_{\lambda_{X}(x)}\right) \subset W_{\lambda_{Y}(y)} .
$$

Consequently,

$$
h(b) \cdot \rho_{Y}(y)=h\left(b \cdot \rho_{X}(x)\right) \in\left(W_{\lambda_{Y}(y)}^{[-1]} V_{y}\right) \cap \lambda_{Y}^{-1}\left(W_{\lambda_{Y}(y)}\right) \subset U_{y}
$$

by the choice of the sets $W_{\lambda_{Y}(y)}$ and $V_{y}$.

By the definition of the set $W_{x} \ni b$, we get $\rho_{Y} \circ h(b)=h \circ \rho_{X}(b) \subset h \circ$ $\rho_{X}\left(W_{x}\right) \subset W_{\rho_{Y}(y)}$. The choice of the neighborhoods $U_{y}$ and $W_{\rho_{Y}(y)}$ guarantees that $h(b) \in\left(U_{y} W_{\rho_{Y}(y)}^{[-1]}\right) \cap \rho_{Y}^{-1}\left(W_{\rho_{Y}(y)}\right) \subset O_{Y}$, which is not possible as $b \in B_{x} \subset B=$ $X \backslash h^{-1}\left(O_{Y}\right)$.

In light of Theorem 3.2 it is important to detect group-refractive topological unosemigroups, in particular, among topological inverse semigroups. Observe that a topological inverse semigroup $S$ is group-refractive if and only if for every non-closed set $B \subset S$ there is a point $x \in \bar{B} \backslash B$ such that for every neighborhood $O_{x x^{-1}} \subset E_{S}$ of the idempotent $x x^{-1}$ there are a subset $B_{x} \subset B$ and an idempotent $e \in O_{x x^{-1}}$ such that $x \in \overline{B_{x}}, e x x^{-1}=e$ and $e B_{x} x^{-1} x \subset H_{e} x$ (which is equivalent to $e B_{x} x^{-1} \subset H_{e}$ ).

\section{Group-refractive topological semilattices}

In this section we shall detect group-refractive topological semilattices. Each semilattice $E$ will be considered as unosemigroup endowed with the identity left and right unit operations $\lambda_{E}(x)=\rho_{E}(x)=x$. 
Each semigroup $E$ is endowed with the partial order $x \leq y$ defined by $x y=x=y x$. For a point $x \in E$ by $\uparrow x=\{y \in E: x \leq y\}$ and $\downarrow x=\{y \in E: y \leq x\}$ we denote its upper and lower cones and by $\Uparrow x$ the interior of $\uparrow x$ in $E$.

A point $x$ of a topological semilattice $E$ will be called locally minimal if its upper cone $\uparrow x$ is open in $E$ and hence coincides with its interior $\Uparrow x$. Observe that a point $x \in E$ is locally minimal if and only if it is isolated in its lower cone $\downarrow x$. By $\mathbb{I}$ we shall denote the unit interval $[0,1]$ endowed with the semilattice operation min : $[0,1] \times[0,1] \rightarrow[0,1]$ of minimum

Rewriting the definition of a group-refractive topological unosemigroup in the case of a topological semilattice and using the continuity of the semilattice operation, we can obtain the following characterization:

Proposition 4.1 A topological semilattice $E$ is group-refractive if and only iffor every non-closed subset $B \subset E$ there is a point $x \in \bar{B} \backslash B$ such that each neighborhood $O_{x} \subset E$ of $x$ contains a point $e \in O_{x}$ such that $x \in \operatorname{cl}_{X}(B \cap \uparrow e)$.

Now we shall study the interplay between the class of group-refractive topological semilattices and some other classes of topological semilattices, defined as follows.

A topological semilattice $E$ is called

- a Lawson semilattice if open subsemilattices form a base of the topology of $E$;

- I-separated if continuous homomorphisms from $E$ to $\mathbb{I}=([0,1]$, min $)$ separate points of $E$;

- a $U$-semilattice if for every open set $U$ in $E$ and point $x \in U$ there is a point $y \in U$ such that $x \in \Uparrow y$;

- a $U_{0}$-semilattice if for every open set $U$ in $E$ and point $x \in U$ there is a (locally minimal) point $y \in U$ such that $x \in \Uparrow y=\uparrow y$;

- a $U_{c l}$-semilattice if for every open set $U$ in $E$, point $x \in U$ and subset $B \subset E$ with $x \in \operatorname{cl}_{E}(B)$ there is a point $y \in U$ such that $x \in \operatorname{cl}_{E}(B \cap \uparrow y)$;

- $U_{c s}$-semilattice if for every open set $U$ in $E$, point $x \in U$ and sequence $\left\{x_{n}\right\}_{n \in \omega} \subset E$ convergent to $x$ there is a point $y \in U$ such that the set $\left\{n \in \omega: x_{n} \in \uparrow y\right\}$ is infinite.

For any Tychonoff topological semilattice these properties relate as follows:

locally compact 0 -dimensional $\Longrightarrow$ locally compact Lawson

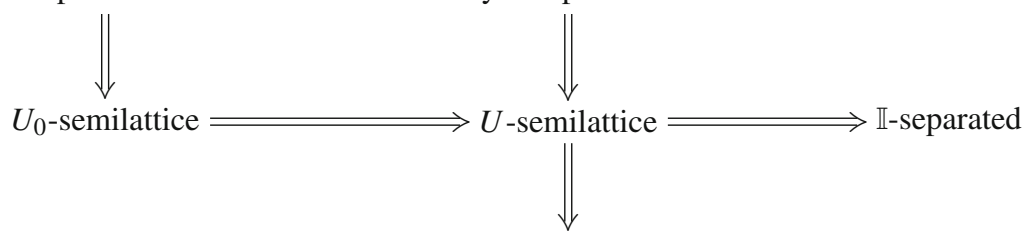

metrizable hereditarily Baire $\Longrightarrow U_{c l}$-semilattice $\Longrightarrow$ group-refractive.

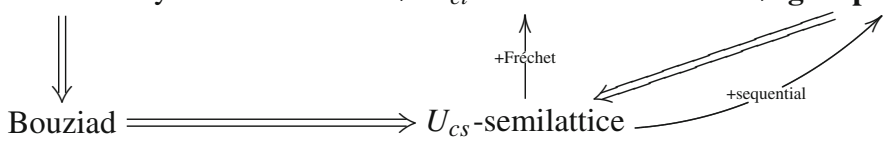

Non-trivial implications from this diagram are proved in the following proposition.

Proposition 4.2 Let E be a Hausdorff topological semilattice. 
(1) If $E$ is locally compact and 0-dimensional, then $E$ is a $V_{0}$-semilattice.

(2) If $E$ is locally compact and Lawson, then $E$ is a $V$-semilattice.

(3) If $E$ is a $U$-semilattice, then $E$ is $\mathbb{I}$-separated.

(4) If $E$ is a $U$-semilattice, then $E$ is a $U_{c l}$-semilattice.

(5) If $E$ is a $U_{c l}$-semilattice, then $E$ is group-refractive.

(6) If $E$ is group-refractive, then $E$ is a $U_{c s}$-semilattice.

(7) If $E$ is a regular Bouziad space, then $E$ is a $U_{c s}$-semilattice.

(8) If $E$ is a metrizable hereditarily Baire space, then $E$ is a $U_{c s}$-semilattice.

(9) If $E$ is a sequential $U_{s c}$-semilattice, then $E$ is group-refractive.

(10) If $E$ is a Fréchet $U_{s c}$-semilattice, then $E$ is a $U_{c l}$-semilattice.

Proof 1-5. The proofs of the first three statements can be found in $[2,2.4]$ and are based on classical results of the theory of topological semilattices, see [5, Ch.2]. The fourth statement is trivial and the fifth statement follows from the definitions.

6. Assume that the topological semilattice $E$ is group-refractive. To prove that $E$ is a $U_{c s}$-semilattice, fix an open set $U \subset E$, a point $x_{\infty} \in U$, and a sequence $\left\{x_{n}\right\}_{n \in \omega}$ convergent to $x_{\infty}$. We need to find a point $y \in U$ such that the set $\left\{n \in \omega: y \leq x_{n}\right\}$ is infinite. If the set $\left\{n \in \omega: x_{n}=x_{\infty}\right\}$ is infinite, then we can put $y=x_{\infty}$ and finish the proof. In the opposite case, we can replace $\left(x_{n}\right)_{n \in \omega}$ by a subsequence and assume that the set $B=\left\{x_{n}\right\}_{n \in \omega}$ does not contain the limit point $x_{\infty}$ and hence $B$ is not closed in $E$. Moreover, since $E$ is Hausdorff, $x_{\infty}$ is a unique point of the set $\bar{B} \backslash B$. By the group-refractivity of $E$ and Proposition 4.1, for the non-closed set $B$ there is a point $x \in \bar{B} \backslash B$ (equal to $x_{\infty}$ ) such that each neighborhood $W_{x} \subset E$ of $x$ contains an idempotent $e \in W_{x}$ with $x_{\infty}=x \in \operatorname{cl}_{X}(B \cap \uparrow e)$. Consequently, the set $\left\{n \in \omega: e \leq x_{n}\right\}=\left\{n \in \omega: x_{n} \in B \cap \uparrow e\right\}$ is infinite, which means that $E$ is a $U_{c s}$-semilattice.

7. Assume that the space $E$ is regular and Bouziad (which means that the player $P$ has no winning strategy in the Bouziad game on $E$ ). To prove that $E$ is a $U_{c s^{-}}$ semilattice, fix an open set $U \subset E$, a point $x \in U$, and a sequence $\left\{x_{k}\right\}_{k \in \omega}$ convergent to $x$. Since the space $X$ is regular, the point $x$ has an open neighborhood $U_{x}$ whose closure is contained in $U$.

Now let us describe a strategy $\$$ of the player $P$ in the Bouziad game on $E$. Let $\tau$ denote the topology of $E$ and $\tau^{<\omega}=\bigcup_{n \in \omega} \tau^{n}$ be the set of all finite sequences $\left(O_{0}, \ldots, O_{n-1}\right)$ of open sets in $E$. The set $\tau^{0}$ is a singleton consisting of the empty sequence and we put $\$_{0}: \tau^{0} \rightarrow\{x\} \subset E$ be the constant function.

By induction, for every $n \in \mathbb{N}$ we can define a function $\$_{n}: \tau^{n} \rightarrow E$ such that for every sequence $\left(O_{0}, \ldots, O_{n-1}\right) \in \tau^{n}$ with $p_{n-1}=\$_{n-1}\left(O_{0}, \ldots, O_{n-2}\right) \in O_{n-1} \cap$ $U_{x} \cap \downarrow x$, the point $p_{n}=\$_{n}\left(O_{0}, \ldots, O_{n-1}\right)$ belongs to the set $O_{n-1} \cap U_{x} \cap \downarrow p_{n-1} \cap \downarrow x_{k_{n}}$ for some $k_{n} \geq n$.

Assume that for some $n \in \mathbb{N}$ the function $\$_{n-1}$ has been constructed. Given any sequence $\left(O_{0}, \ldots, O_{n-1}\right) \in \tau^{n}$, put $\$_{n}\left(O_{0}, \ldots, O_{n-1}\right)=x$ if $p_{n-1}=$ $\$_{n-1}\left(O_{0}, \ldots, O_{n-2}\right) \notin O_{n-1} \cap U_{x} \cap \downarrow x$. In the opposite case, the point $p_{n-1}$ belongs to $O_{n-1} \cap U_{x} \cap \downarrow x$ and by the continuity of the semilattice operation at $p_{n-1}=p_{n-1} x$, we can find a neighborhood $W_{x} \subset E$ of $x$ such that $p_{n-1} W_{x} \subset O_{n-1} \cap U_{x}$. By the convergence of the sequence $\left(x_{k}\right)_{k \in \omega}$ to $x$, there is a number $k_{n} \geq n$ such that $x_{k_{n}} \in W_{x}$. Then the point $p_{n}=p_{n-1} x_{k_{n}}$ belongs to the set $O_{n-1} \cap U_{x} \cap \downarrow p_{n-1} \cap \downarrow x_{k_{n}}$ and 
we can put $\$_{n}\left(O_{0} \ldots, O_{n-1}\right):=p_{n}$. This completes the definition of the function $\$_{n}: \tau^{n} \rightarrow E$.

The function $\$=\bigcup_{n \in \omega} \$_{n}: \tau^{<\omega} \rightarrow E$ can be considered as a strategy of the player $P$ in the Bouziad game on the space $E$. By our assumption, this strategy is not winning, which means that there is an infinite sequence $\left(O_{n}\right)_{n \in \omega}$ of open sets of $E$ such that each set $O_{n}$ is a neighborhood of the point $p_{n}=\$_{n}\left(O_{0}, \ldots, O_{n-1}\right)$ and the sequence $\left(p_{n}\right)_{n \in \omega}$ has an accumulation point $p_{\infty}$ in $E$. Using the fact that $p_{0}=x \in O_{0} \cap U_{x} \cap \downarrow x$, by induction on $n \in \omega$ we can show that $p_{n-1} \in O_{n-1} \cap U_{x} \cap \downarrow x$ and $p_{n} \in$ $O_{n-1} \cap U_{x} \cap \downarrow p_{n-1} \cap \downarrow x_{k_{n}}$. This means that the sequence $\left(p_{n}\right)_{n \in \omega}$ is decreasing and by the continuity of the semilattice operation, $p_{\infty} \leq p_{n} \leq p_{n-1} x_{k_{n}} \leq x$ for all $n \in \mathbb{N}$. So, $e=p_{\infty} \in \operatorname{cl}_{E}\left(U_{x}\right) \subset U$ is a point such that the set $\left\{k \in \omega: e \leq x_{k}\right\} \supset\left\{k_{n}\right\}_{n \in \omega}$ is infinite, witnessing that $E$ is a $U_{c S}$-semilattice.

8. The eighth statement follows from Propositions 4.2(7) and 2.6.

9. The ninth statement follows trivially from Proposition 4.1 .

10. Assume that $E$ is a Fréchet $U_{c s}$-semilattice. To prove that $E$ is a $U_{c l}$-semilattice, fix any open set $U \subset E$, point $x \in U$ and a subset $B \subset E$ containing the point $x$ in its closure. Since the space $E$ is Fréchet, some sequence $\left\{x_{n}\right\}_{n \in \omega} \subset B$ converges to $x$. Since $E$ is a $U_{c s}$-semilattice, there is a point $y \in U$ such that the set $\left\{n \in \omega: y \leq x_{n}\right\}$ is infinite and hence the set $B \cap \uparrow y \subset\left\{x_{n}: n \in N\right\}$ contains $x$ in its closure. This means that $E$ is a $U_{c l}$-semilattice.

Proposition 4.2(6,9) implies the following characterization of sequential grouprefractive topological semilattices.

Corollary 4.3 A Hausdorff sequential topological semilattice is group-refractive if and only if it is a $U_{c s}$-semilattice.

\section{Group-refractive topological inverse semigroups}

In this section we apply the results on group-refractive topological semilattices to derive some criteria of group-refractivity of topological inverse and Clifford semigroups.

Proposition 5.1 A topological inverse semigroup $S$ is group-refractive if its maximal semilattice $E_{S}$ is a $U_{0}$-semilattice.

Proof Assume that $E$ is a $U_{0}$-semilattice. To prove that $S$ is group-refractive, take any non-closed subset $B \subset S$ and fix any point $x \in \bar{B} \backslash B$. Given any neighborhood $W_{x x^{-1}} \subset \lambda_{S}(S)=E_{S}$ of the idempotent $x x^{-1}=\lambda_{S}(x)$, we need to find an idempotent $e \in W_{x x^{-1}}$ and a subset $B_{x} \subset B$ such that $x \in \operatorname{cl}_{S}\left(B_{x}\right), e \leq x x^{-1}$, and $e B_{x} \rho_{S}(x) \subset$ $H_{e} x$ (which is equivalent to $e B_{x} x^{-1} \subset H_{e}$ ).

Since $E$ is a $U_{0}$-semilattice, there is an idempotent $e \in W_{x x^{-1}}$ such that $x x^{-1} \in$ $\uparrow e=\Uparrow e$. Since the upper cone $\uparrow e=\Uparrow e$ is open in $E_{S}$, we can find an open set $W \subset S$ such that $W \cap E_{S}=\Uparrow e$. Taking into account that $x x^{-1} x x^{-1}=x x^{-1} \in \Uparrow e \subset W$ and $x x^{-1} e x x^{-1}=e \in W$, by the continuity of the algebraic operations on $S$, we can find a neighborhood $V \subset S$ of $x$ such that $\left(V V^{-1} V V^{-1}\right) \cup\left(e V V^{-1} V V^{-1}\right) \cup$ $\left(V V^{-1} e V V^{-1}\right) \subset W$. Since the set $B_{x}=B \cap V$ contains $x$ in its closure and 
$e \leq x x^{-1}=\lambda_{S}(x)$, to finish the proof it remains to show that $e B_{x} x^{-1} \subset H_{e}$. Given any point $b \in B_{x} \subset V$, we need to check that $\left(e b x^{-1}\right)\left(e b x^{-1}\right)^{-1}=e=$ $\left(e b x^{-1}\right)^{-1}\left(e b x^{-1}\right)$. The first equality follows from the inclusion $e b x^{-1} x b^{-1} e^{-1}=$ $e b x^{-1} x b^{-1} \in E_{S} \cap\left(e V V^{-1} V V^{-1}\right) \subset E_{S} \cap W=\Uparrow e$.

The inequality $x b^{-1} e b x^{-1} \geq e$ (which is a part of the second equality) follows from the inclusion $x b^{-1} e b x^{-1} \in E_{S} \cap\left(V V^{-1} e V V^{-1}\right) \subset E_{S} \cap W=\uparrow e$. By analogy we can prove that $b x^{-1} e x b^{-1} \in E_{S} \cap\left(V V^{-1} e V V^{-1}\right) \subset E_{S} \cap W=\uparrow e$, which implies that

$$
\left(b x^{-1}\right)^{-1} b x^{-1} e x b^{-1}\left(b x^{-1}\right) \geq\left(b x^{-1}\right)^{-1} e b x^{-1} .
$$

Since $x b^{-1} b x^{-1} \in E_{S} \cap\left(V V^{-1} V V^{-1}\right) \subset \uparrow e$, we conclude that $e=x b^{-1} b x^{-1} e x b^{-1}$ $b x^{-1} \geq x b^{-1} e b x^{-1}$. So, $x b^{-1} e b x^{-1}=e$ and hence $e b x^{-1} \in H_{e}$ and $e b \cdot \rho_{S}(x)=$ $e b x^{-1} x \in H_{e} x$.

For topological Clifford semigroups this proposition can be enforced as follows.

Proposition 5.2 A topological Clifford semigroup $S$ is group-refractive if its maximal semilattice $E_{S}$ is a $U$-semilattice.

Proof To prove that $S$ is a group-refractive, fix any non closed subset $B \subset S$ and choose any point $x \in \bar{B} \backslash B$. Since $S$ is a Clifford semigroup, the left and right unit operations $\lambda_{S}$ and $\rho_{S}$ coincide with the homomorphism $\pi: S \rightarrow E_{S}, \pi: x \mapsto x x^{-1}=x^{-1} x$.

Assuming that $E_{S}$ is a $U$-semilattice, for every neighborhood $W_{\lambda_{S}(x)} \subset E_{S}$ of the idempotent $\lambda_{S}(x)=\pi(x)$ we can find an idempotent $e \in W_{\lambda_{S}(x)}$ such that $\pi(x) \in \Uparrow e$. Since $\pi^{-1}(\Uparrow e)$ is an open neighborhood of the point $x \in \operatorname{cl}_{S}(B)$, the set $B_{x}=\pi^{-1}(\Uparrow e) \cap B$ contains $x$ in its closure.

It remains to check that $e \cdot B_{x} \cdot \rho_{S}(x) \subset H_{e} x$. First we show that $H_{e} \subset H_{e} x$. For this observe that $\pi\left(H_{e} x^{-1}\right)=\left\{e \pi\left(x^{-1}\right)\right\}=\left\{e x x^{-1}\right\}=\{e\}$ and hence $H_{e} x^{-1} \subset$ $\pi^{-1}(e)=H_{e}$. Multiplying the inclusion $H_{e} x^{-1} \subset H_{e}$ by $x$ from the right, we get $H_{e} x^{-1} x \subset H_{e} x$ and hence $H_{e}=H_{e} e=H_{e} e x^{-1} x=H_{e} x^{-1} x \subset H_{e} x$.

Since idempotents of any Clifford semigroup lie in its center [10, II.2.6], we get $e \cdot B_{x} \cdot \rho_{S}(x)=e B_{x} x^{-1} x=e x^{-1} x B_{x}=e x x^{-1} B_{x}=e B_{x}$ and hence $\pi\left(e \cdot B_{x}\right.$. $\left.\rho_{S}(x)\right)=\pi\left(e B_{x}\right)=\pi(e) \cdot \pi\left(B_{x}\right) \subset e \cdot \Uparrow e=\{e\}$, which yields the desired inclusion

$$
e \cdot B_{x} \cdot \rho_{S}(x) \subset \pi^{-1}(e)=H_{e} \subset H_{e} x
$$

Proposition 5.3 For a Hausdorff sequential topological Clifford semigroup $S$ and its maximal semilattice $E_{S}$ the following conditions are equivalent:

(1) $S$ is group-refractive;

(2) $E_{S}$ is group-refractive;

(3) $E_{S}$ is a $U_{c s}$-semilattice.

Proof The implication (1) $\Rightarrow(2)$ is trivial and (2) $\Rightarrow$ (3) was proved in Proposition 4.2(6). 
To prove the implications (3) $\Rightarrow(1)$, take any non-closed subset $B \subset S$ and by the sequentiality of $S$, find a sequence $\left\{x_{n}\right\}_{n \in \omega}$, convergent to a point $x \in \bar{B} \backslash B$. Given any neighborhood $W_{x x^{-1}} \subset E_{S}$ of the idempotent $x x^{-1}=\lambda_{S}(S)$, we need to find an idempotent $e \in W_{x x^{-1}} \cap \downarrow x x^{-1}$ and a subset $B_{x} \subset B$ such that $x \in \operatorname{cl}\left(B_{x}\right)$ and $e B_{x} x^{-1} \subset H_{e}$.

Consider the continuous homomorphism $\pi=\lambda_{S}=\rho_{S}: S \rightarrow E_{S}, \pi: x \mapsto$ $x x^{-1}=x^{-1} x$, and observe that the sequence of idempotents $e_{n}=\pi\left(x_{n}\right)$ converges to the idempotent $\pi(x) \in W_{x x^{-1}}$. Since $E$ is a $U_{c s}$-semilattice, there is an idempotent $e \in W_{x x^{-1}}$ such that the set $N=\left\{n \in \omega: e \leq e_{n}\right\}$ is infinite and hence $\pi(x)=$ $\lim _{N \ni n \rightarrow \infty} \pi\left(x_{n}\right)=\lim _{N \ni n \rightarrow \infty} e_{n} \geq e$. Then the subset $B_{x}=\left\{x_{n}: n \in N\right\} \subset B$ contains the point $x$ in its closure and for every $n \in N$

$$
\pi\left(e x_{n} x^{-1}\right)=\pi(e) \pi\left(x_{n}\right) \pi\left(x^{-1}\right)=e e_{n} \pi(x)=e,
$$

which implies $e x_{n} x^{-1} \in \pi^{-1}(e)=H_{e}$ and $e B_{x} x^{-1} \subset H_{e}$.

Propositions 5.3 and 4.2(7,8) imply:

Corollary 5.4 A sequential Hausdorff topological Clifford semigroup $S$ is grouprefractive, if its maximal semilattice $E_{X}$ is metrizable and hereditarily Baire (more generally, regular and Bouziad).

Corollary 5.5 A metrizable topological Clifford semigroup $S$ with hereditarily Baire maximal semilattice $E_{S}$ is group-refractive.

\section{The continuity of $E H$-continuous homomorphisms between topological inverse semigroups}

In this section we establish some criteria of the continuity of $E H$-continuous homomorphisms between topological inverse semigroups.

Theorem 6.1 Each EH-continuous homomorphism $h: X \rightarrow Y$ from a grouprefractive topological inverse semigroup $X$ into a ditopological inverse semigroup $Y$ is continuous.

Proof The group-refractive topological inverse semigroup $X$ endowed with the canonical left and right unit operations is a group-refractive $\lambda$-regular topological unosemigroup, and the ditopological inverse semigroup $Y$ is a ditopological unosemigroup. Since each homomorphism between inverse semigroups preserves the operation of inversion, it also preserves the left and right unit operations, and hence is a unomorphism between the corresponding unosemigroups. So, $h: X \rightarrow Y$ is a unomorphism and by Theorem 3.2, it is continuous, being $E H$-continuous.

Combining Theorem 6.1 with Proposition 5.1, we get:

Corollary 6.2 If the maximal semilattice $E_{X}$ of a topological inverse semigroup $X$ is a $U_{0}$-semilattice, then each $E H$-continuous homomorphism $h: X \rightarrow Y$ into a ditopological inverse semigroup $Y$ is continuous. 
Since each Hausdorff locally compact zero-dimensional topological semilattice is a $U_{0}$-semilattice (see Proposition 4.2(1)), Corollary 6.2 implies:

Corollary 6.3 If the maximal semilattice $E_{X}$ of a topological inverse semigroup $X$ is Hausdorff, locally compact and zero-dimensional, then each E H-continuous homomorphism $h: X \rightarrow Y$ into a ditopological inverse semigroup $Y$ is continuous.

Since each compact Hausdorff topological inverse semigroup is ditopological (see Proposition 2.2), Corollary 6.3 implies:

Corollary 6.4 If the maximal semilattice $E_{X}$ of a topological inverse semigroup $X$ is Hausdorff, locally compact and zero-dimensional, then each E H-continuous homomorphism $h: X \rightarrow Y$ into a compact Hausdorff topological inverse semigroup $Y$ is continuous.

\section{The continuity of $\boldsymbol{E} \boldsymbol{H}$-continuous homomorphisms between topological Clifford semigroups}

In this section we establish some criteria of the continuity of $E H$-continuous homomorphisms between topological Clifford semigroups. Combining Theorem 6.1 with Proposition 5.2 we get the following theorem, which generalizes an old result of Bowman [4].

Theorem 7.1 If the maximal semilattice $E_{X}$ of a topological Clifford semigroup $X$ is a $U$-semilattice, then each EH-continuous homomorphism $h: X \rightarrow Y$ to a ditopological inverse semigroup $Y$ is continuous.

Since each Hausdorff locally compact Lawson semilattice is a $U$-semilattice (see Proposition 4.2(2)), Theorem 7.1 implies:

Corollary 7.2 If the maximal semilattice $E_{X}$ of a topological Clifford semigroup $X$ is Hausdorff, locally compact and Lawson, then each E H-continuous homomorphism $h: X \rightarrow Y$ to a ditopological inverse semigroup $Y$ is continuous.

Combining Theorem 6.1 with (the proof of) Proposition 5.3, we get:

Corollary 7.3 If the maximal semilattice $E_{X}$ of a sequential topological Clifford semigroup $X$ is a $U_{c s}$-semilattice, then each $E H$-continuous homomorphism $h: X \rightarrow$ $Y$ to a ditopological inverse semigroup $Y$ is continuous.

Since each regular Bouziad topological semilattice is a $U_{c s}$-semilattice (see Proposition 4.2(7)), Corollary 7.3 implies:

Corollary 7.4 If the maximal semilattice $E_{X}$ of a sequential topological Clifford semigroup $X$ is regular and Bouziad, then each $E H$-continuous homomorphism $h$ : $X \rightarrow Y$ to a ditopological inverse semigroup $Y$ is continuous.

Since each Čech-complete space is Bouziad, Corollary 7.4 implies: 
Corollary 7.5 Each EH-continuous homomorphism $h: X \rightarrow Y$ from a sequential Čech-complete topological Clifford semigroup $X$ to a ditopological inverse semigroup $Y$ is continuous.

Since each metrizable hereditarily Baire space is Bouziad, Corollary 7.4 implies:

Corollary 7.6 Each EH-continuous homomorphism $h: X \rightarrow Y$ from a metrizable hereditarily Baire topological Clifford semigroup $X$ to a ditopological inverse semigroup $Y$ is continuous.

Since each compact Hausdorff topological inverse semigroup is ditopological (see Proposition 2.2), Corollaries 7.5 and 7.6 imply:

Corollary 7.7 Each EH-continuous homomorphism $h: X \rightarrow Y$ from a sequential Čech-complete topological Clifford semigroup X to a compact Hausdorff topological inverse semigroup $Y$ is continuous.

Corollary 7.8 Each EH-continuous homomorphism $h: X \rightarrow Y$ from a metrizable hereditarily Baire topological Clifford semigroup $X$ to a compact Hausdorff inverse semigroup $Y$ is continuous.

\section{Open problems}

We do not know if the requirement of sequentiality can be removed from Corollary 7.7.

Problem 8.1 Is each $E H$-continuous homomorphism $h: X \rightarrow Y$ from a Čechcomplete topological Clifford semigroup $X$ into a compact Hausdorff topological Clifford semigroup $Y$ continuous?

Another open problem asks if Yeager's Theorem 1.1 still remains true for homomorphisms between compact topological inverse semigroups.

Problem 8.2 Let $h: X \rightarrow Y$ be a $E H$-continuous homomorphism between compact Hausdorff topological inverse semigroups (with Lawson maximal semilattices). Is $h$ continuous?

Acknowledgments The authors have been partially financed by NCN Grant DEC-2012/07/D/ST1/02087.

Note added in proof I. Pastukhova recently proved the continuity of any EH-continuous homomorphism between compact topological inverse semigroups with Lawson maximal semilattices.

Open Access This article is distributed under the terms of the Creative Commons Attribution License which permits any use, distribution, and reproduction in any medium, provided the original author(s) and the source are credited.

\section{References}

1. Banakh, T., Pastukhova, I.: Topological and ditopological unosemigroups. Mat. Stud. 39(2), 119-133 (2013) 
2. Banakh, T, Pastukhova, I.: On topological Clifford semigroups embeddable into products of cones over topological groups. Semigr. Forum 89(2), 367-382 (2014) http://arxiv.org/abs/1304.2558

3. Bouziad, A.: The Ellis theorem and continuity in groups. Topol. Appl. 50, 73-80 (1993)

4. Bowman, T.: A construction principle and compact Clifford semigroups. Semigr. Forum 2(4), 343-353 (1971)

5. Carruth, J.H., Hildebrant, J.A., Koch, R.J.: The Theory of Topological Semigroups, vol. 2. Marcel Dekker Inc, New York (1986)

6. Choquet, G.: Lectures on Analysis I. Benjamin, New York (1969)

7. Debs, G.: Espaces héréditairement de Baire. Fund. Math. 129, 199-206 (1988)

8. Engelking, R.: General Topology. Heldermann, Berlin (1989)

9. Hurewicz, W.: Relativ perfekte Teile von Punktmengen und Mengen (A). Fund. Math. 12, 78-109 (1928)

10. Petrich, M.: Inverse Semigroups. Wiley, New York (1984)

11. Telgársky, R.: Remarks on a game of Choquet. Colloq. Math. 51, 365372 (1987)

12. Telgársky, R.: Topological games: on the 50th anniversary of the Banach-Mazur game. Rocky Mt. J. Math. 17(2), 227-276 (1987)

13. Yeager, D.P.: On the topology of a compact inverse Clifford semigroup. Trans. Am. Math. Soc. 215, 253-267 (1976) 